\title{
Bronchial Necrosis from Mucormycosis: A Case Report and Review
}

\author{
Jessica Heimes $^{1 *}$, Salman Zaheer ${ }^{1}$ and Jason Wallen ${ }^{2}$
}

${ }^{1}$ Department of Thoracic Surgery, Loma Linda University, Loma Linda, CA, USA

${ }^{2}$ Department of Thoracic Surgery, Upstate University, Syracuse, NY, USA

\begin{abstract}
Disseminated pulmonary mucormycosis is an uncommon and aggressive life threatening condition associated with a high mortality rate. Pulmonary mucormycosis can spread to contralateral lung before it is even detected, increasing mortality rate with little chance of cure. Our report describes an aggressive case of disseminated mucormycosis, involving bronchial necrosis leading to a bilobectomy with tissue flap coverage. Although our report describes a fatal outcome, this case evaluates the role for surgery in the treatment of difficult infections.
\end{abstract}

Keywords: Pulmonary mucormycosis; Bronchial necrosis

\section{Introduction}

Mucormycosis is a lethal infection among immunocompromised hosts. Pulmonary mucormycosis can spread to contralateral lung before it is even detected. We present a case of pulmonary and disseminated mucormycosis in a young diabetic female after a tooth extraction.

\section{Case Report}

A 22-year-old female was admitted to an outside hospital after she was found unresponsive in her home. She was taken to an outside hospital where she was found to have diabetic ketoacidosis, septic shock, acute respiratory failure, and acute renal failure. Two days prior to her admission she underwent a tooth extraction. She began complaining of fevers, chills, headaches, changes in vision, cough, shortness of breath, chest pain, abdominal pain, nausea, vomiting, diarrhea, and a rash soon after this procedure. She did not present to a physician until her significant other was unable to awake her.

Her past medical history is significant for poorly controlled diabetes mellitus type I, with recent Hemoglobin A1c of greater than 14 percent and occasional methamphetamine use. She has had no prior operations nor other significant family or social history.

Upon admission at the outside hospital she was found to be in septic shock with lactate of $11 \mathrm{mMol} / \mathrm{L}$, diabetic ketoacidosis, acute respiratory failure requiring mechanical ventilation, and acute renal failure. After cultures were obtained, antibiotics were started. A CT scan was performed on the abdomen and pelvis revealing pneumatosis intestinalis and pneumobilia. General surgery was involved and no acute intervention was pursued as her exam was benign. She improved and was extubated after a few days. Later the same day, she had a cardiac arrest and was re-intubated. An echocardiogram was done revealing an ejection fraction of 65-75 percent with mild tricuspid and pulmonary valve regurgitation. A bronchoscopy was performed concerning for necrotizing infection of the right middle and lower lobes. A CT scan of the chest was performed, demonstrating diffuse necrosis of the right lower lobe and peri-bronchiolar necrosis of the right middle lobe (Figure 1). Blood cultures produced positive results for Escherichia coli and Candida glabrata. A sputum culture produced an unspecified organism, concerning for mucormycosis. She was transferred to our institution for further care.

Upon transfer, she remained intubated, on 40 percent oxygen with saturations measuring 99 percent. She was awake with a residual right sided foot drop after her recent cardiac arrest. Her white blood cell count was $10.6 \mathrm{bil} / \mathrm{L}$ on admission with hemoglobin $9.6 \mathrm{~g} / \mathrm{dL}$, Creatinine $0.6 \mathrm{mg} / \mathrm{dL}$, albumin $2.9 \mathrm{~g} / \mathrm{dL}$, and transthyretin $8.6 \mathrm{mg} / \mathrm{dL}$. HIV rapid screen was negative. Other labs were within normal limits. Her vital

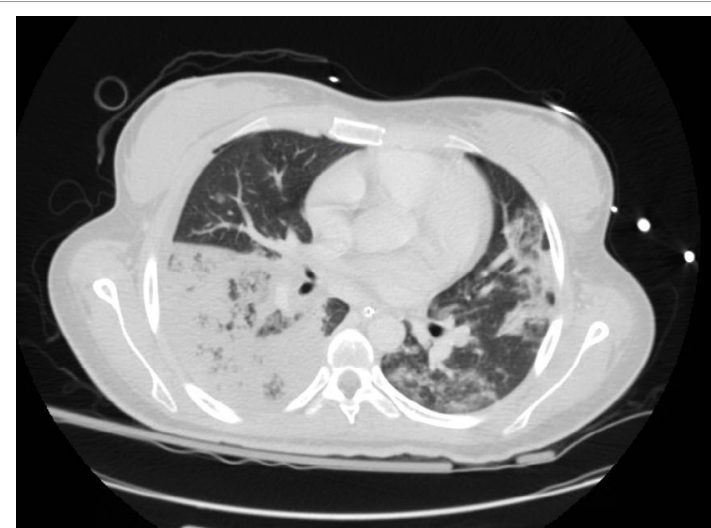

Figure 1: CT scan of the chest demonstrating diffuse necrosis of the right lower lobe and peri-bronchiolar necrosis of the right middle lobe.

signs were within normal limits for her age and she did not require vasopressor support. A repeat CT scan of the head, chest, abdomen, and pelvis was performed. The CT scan of the chest revealed increasing opacities and the presence of a small pneumothorax (Figure 2). The CT scan of the abdomen revealed a loculated fluid collection measuring 9 $\mathrm{cm} \times 7 \mathrm{~cm}$, concerning for an abscess and a small lesion in the spleen concerning for infarct or abscess. The CT scan of the head showed mild ventricular predominance, concerning for early communicating hydrocephalus.

Infectious disease, pulmonary medicine, thoracic surgery, and critical care physicians were involved with her care. She was given supportive measures, including Intravenous fluids, insulin, nutrition, antibiotics, and antifungals. She was given piperacillin-tazobactam $4.5 \mathrm{~g}$ intravenous every $6 \mathrm{~h}$, vancomycin $1000 \mathrm{mg}$ intravenous every $12 \mathrm{~h}$, and voriconazole $200 \mathrm{mg}$ intravenous every $12 \mathrm{~h}$. Voriconazole was started because initial fungal cultures showed Candida krusei and Aspergillus species, not A. fumigatus. After further discussion with

*Corresponding authors: Jessica Heimes, Department of Thoracic Surgery Loma Linda University, 11234 Anderson St. Schuman Pavilion, Suite 1617, Loma Linda, CA, USA, Tel: 909-558-4354; Fax: 909-558-0348; E-mail: jheimes@llu.edu

Received December 04, 2016; Accepted December 30, 2016; Published December 31, 2016

Citation: Heimes J, Zaheer S, Wallen J (2016) Bronchial Necrosis from Mucormycosis: A Case Report and Review. J Pulm Respir Med 6: 385. doi: 10.4172/2161-105X.1000385

Copyright: $\odot 2016$ Heimes J, et al. This is an open-access article distributed under the terms of the Creative Commons Attribution License, which permits unrestricted use, distribution, and reproduction in any medium, provided the original author and source are credited. 


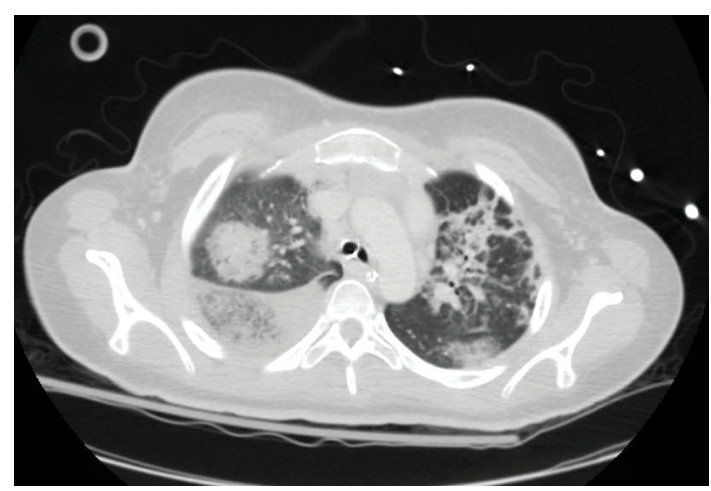

Figure 2: CT scan of the chest revealed increasing opacities and the presence of a small pneumothorax.

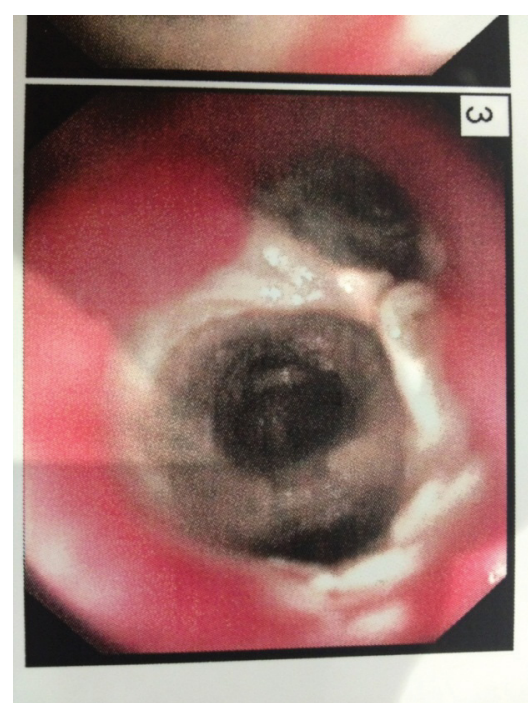

Figure 3: Bronchial necrosis of the right middle and lower lobes.

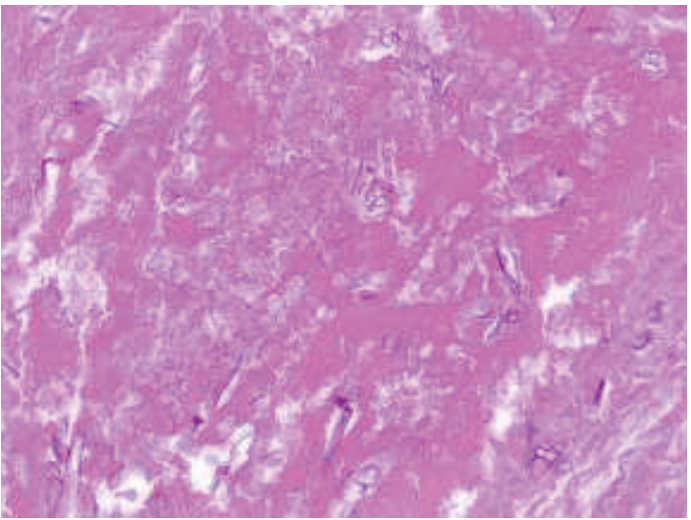

Figure 4: Broad based hyphae in the lung vasculature and parenchyma.

the patient, the decision was made to take the patient to the operating room for debridement of the necrotizing pneumonia and likely invasive mucormycosis.

A bronchoscopy, right lower bilobectomy, and intercostal muscle flap over the bronchial stump was performed. Intraoperatively pictures were taken of the right middle and lower lobes, showing bronchial necrosis (Figure 3 ). The lower lobe parenchyma was severely diseased with patchy involvement of the middle lobe parenchyma was present. After resection bronchoscopy was performed and the bronchial stump appeared viable at the staple line. This bronchial staple line was covered with an intercostal pedicled muscle flap.

The specimen was sent to pathology for evaluation and the patient had invasive mucormycosis, involving the vascular margins, with extensive parenchymal necrosis and hemorrhage. Broad based hyphae were found in the lung vasculature and parenchyma (Figure 4). Fungal cultures had been sent but were negative for growth after 4 weeks.

She was extubated on postoperative day 2 and received aggressive pulmonary treatments. Antifungal medications were adjusted and the patient was started on liposomal amphotericin B at $10 \mathrm{mg} / \mathrm{kg}$ intravenous every $24 \mathrm{~h}$, caspofungin $100 \mathrm{mg}$ intravenous every $24 \mathrm{~h}$, and posaconazole $300 \mathrm{mg}$ intravenous every $12 \mathrm{~h}$. Ophthalmology and ENT were consulted for dilated eye exam and exam for invasive Mucor to the sinuses and Candida glabrata. No evidence of invasive Mucor was identified in these areas. Interventional radiology drained the pelvic fluid collection and cultures were sent with no evidence of mucormycosis. She was unable to swallow without aspiration after extubation and a nasojejunal feeding tube was placed for nutrition. Her chest tubes were removed on postoperative day 6 . She was reintubated for respiratory failure on postoperative day 12 and was unable to be weaned from the ventilator. A percutaneous tracheostomy was placed on postoperative day 16 for prolonged intubation. On postoperative day 21 she arrested and was given multiple rounds of epinephrine and chest compressions. She was resuscitated but air and fluid was seen coming from her chest tube sites. Her tracheostomy tube was exchanged over a bronchoscope for an endotracheal tube which was placed in the left mainstem bronchus for exclusion of the right lung. Upon evaluation of the right bronchial stump, a large bronchopleural fistula was identified. A chest tube was replaced on the right side for drainage of air and fluid.

After further discussions with her family and the poor prognosis associated with her disease, the family decided to move to comfort care and withdraw life support on postoperative 22 . The patient expired and no autopsy was performed.

\section{Discussion}

Mucormycosis is a rare fungal infection from Rhizopus, Mucor, Lichtheimia, Cunninghamella berthollethiae species [1,2]. The spores are generally inhaled or enter through open wounds and then germinate in the host forming hyphal elements [1-4]. Rhinocerebral and pulmonary forms are the most common manifestations of mucormycosis encountered at 55 and 30 percent respectively [5]. Disseminated mucormycosis occurs in 9 percent of cases [5]. Pulmonary mucormycosis infections usually occur in patients who are immunocompromised, such as transplant recipients (especially lung and liver transplant patients), chemotherapy patients, chronic corticosteroid therapy, renal failure, or even diabetics [2].

In healthy individuals, mononuclear and polymorphonuclear phagocytes eliminate fungal spores and hyphae by oxidative and nonoxidative killing mechanisms [2,6,7]. Defects in phagocytic cell activity permit unrestricted growth of the hyphal form and lead to invasive infection, such as those seen with hyperglycemia and acidosis $[2,6-8]$. Ibrahim et al. [9] discovered a glucose regulated protein 78 (GRP78) as a novel host receptor that interacts with Rhizopus, mediating invasion and damage of human endothelial cells by the fungus $[2,10]$. Elevated glucose and iron levels upregulate GRP78 expression and promote endothelial cell invasion and damage [2]. The immunosuppressed patient has an increased risk of infection with Mucor species. 
Corticosteroids impair migration, ingestion, and phagolysosome fusion in human macrophages, increasing the susceptibility to Mucor infections $[2,6,7]$. Patients in iron overload states, including those patients undergoing chelation therapy with deferoxamine are predisposed to Mucor infections. Deferoxamine eliminates the fungistatic effect of serum and increases in vitro fungal growth by acting as a siderophore for Mucorales species [2,11-13]. Iron chelators such as deferiprone and deferasirox which lack xenosiderophore activity and have been shown to be protective in animal models of mucormycosis $[2,12]$. Some case reports show a potential beneficial effect of deferasirox therapy in Mucor patients [2,14]. Diabetic individuals have a tendency for increased serum iron secondary to impaired transferrin binding, leading to increased infection [4].

Pulmonary mucormycosis is the second most common presentation of Mucor in diabetics, while the rhinocerebral form is most common in this population [4]. Mucor species flourish in acidic environments, therefore renal failure and diabetics have increased susceptibility [1]. In addition, this species has a ketone reductase enzyme that allows it to thrive in high glucose environments [4]. Prolonged treatments with voriconazole has been associated with an increased incidence of these infections because it is not active against these fungi $[3,4]$.

Mucor hyphae are well known to be angioinvasive, causing hemorrhage, thrombosis, infarction, and tissue necrosis of invaded tissues. Pulmonary mucormycosis spreads rapidly when the spores spread into bronchioles and alveoli, causing pulmonary parenchymal necrosis and a high mortality rate [1]. Endobronchial lesions are found in a third of pulmonary mucormycosis patients leading to high mortality rates secondary to obstruction of the airways and vascular invasion leading to hemorrhage [4]. The in-hospital mortality rate with isolated pulmonary mucormycosis is 65 percent. If the disease is disseminated the mortality rate reaches 98 percent [1]. The most common cause of death is fungal sepsis related to disseminated disease. Mucor can spread rapidly to the contralateral lung and distal organs when treatment is not started in a timely fashion $[2,15]$. Pulmonary mucormycosis patients usually die from disseminated disease before respiratory failure occurs. However, dissemination is rarely detected antemortem $[2,15,16]$.

High resolution CT scan is the best method of determining the extent of pulmonary mucormycosis and typically shows evidence of infection earlier than standard chest radiographs [2]. Nodular opacities without an air bronchogram are the most common findings on CT scan and may be accompanied by multiple nodules or pleural effusions $[2,17,18]$. Halo and air-crescent signs are associated with mucormycosis, but in centrally located lesions there is increased risk of pulmonary artery erosion and hemoptysis [2]. Mucormycosis has been associated with a reverse halo sign, described as a focal round area of ground-glass attenuation surrounded by a ring consolidation $[2,16,17]$.

Diagnosis is difficult in mucormycosis because tissue swabs, sputum, and bronchial alveolar lavage fluid are usually nondiagnostic $[2,19]$. Despite invasion of blood vessels, blood cultures rarely grow Mucorales species [2]. Mucorales appear as broad nonseptate hyphae with branches occurring at right angles [20].

Mucormycosis is treated with antifungals, surgery, reducing immunosuppression, control of blood glucose, and discontinuation of deferoxamine treatment $[2,13,21]$. Amphotericin B is the initial treatment of choice and is the only approved drug for the treatment of mucormycosis $[3,4]$. The largest reported case series of mucormycosis included 24 patients who received a lipid complex of amphotericin B with overall response rate of 71 percent without significant toxic effects, even in those with pre-existing renal disease $[2,22]$. Doses have been suggested from $5 \mathrm{mg} / \mathrm{kg} /$ day to $10 \mathrm{mg} / \mathrm{kg} /$ day up to $10 \mathrm{mg} / \mathrm{kg} /$ day to $15 \mathrm{mg} / \mathrm{kg} /$ day [2-4,23-25]. Further trials are ongoing to determine appropriate doses of amphotericin B.

Posaconazole may be an alternative therapy for intolerance, or used in conjunction with amphotericin B [3]. The dosage of posaconazole was $800 \mathrm{mg}$ daily in four divided doses which showed an overall success rate of 70 percent in 24 patients with minimal toxicity [2,2628]. A retrospective review of posaconazole based salvage therapy in 91 patients with refractory mucormycosis revealed an overall success rate of 61 percent $[2,29]$. Posaconazole is an oral medication with limitations associated with absorption, especially those with poor oral intake $[2,7,8]$. The steady-state plasma concentrations of the drug are not reached until 1 week of therapy has started $[2,7,8]$. However, the data is not complete. Some studies have found combination therapy beneficial while others have found superior results with amphotericin monotherapy [5]. Antifungals such as voriconazole and caspofungin are not effective against Mucor and prophylaxis with these drugs has been shown to be associated with Mucor infections [4]. However, a combination of amphotericin B and caspofungin was associated with improved therapy in 41 patients with biopsy-proven rhinoorbital cerebral mucormycosis $[2,30]$. Duration of therapy should be individualized.

Surgical debridement is indicated in patients with isolated mucormycosis involving one lung and should be performed early in those who can tolerate the procedure [1,31]. Antifungal agents usually have poor penetration at the site of infection secondary to thrombosis and tissue necrosis, therefore surgical debridement is of paramount importance [2]. Tedder et al. [31] found a benefit to aggressive surgical resection in pulmonary mucormycosis. They found a 9.4 percent mortality rate in patients treated with surgical resection compared to 50 percent mortality in those treated with medical therapy alone [1,31]. An additional review found the mortality rate in those who received antifungal therapy alone for pulmonary mucormycosis was 55 percent, compared with those who received both antifungals and surgery $[2,15]$. Lobectomy, and sometimes pneumonectomy, may be required for improvement. The benefit of pulmonary resection decreases after the disease disseminates.

Other treatments have been proposed to combat Mucorales. One such therapy mentioned in the literature include hyperbaric oxygen, especially for rhinocerebral disease [2,32]. Cytokine therapy using interferon gamma and granulocyte-macrophage-colony stimulating factor have been proposed to improve the ability of phagocytic cells to kill Mucorales species $[2,6-8,13]$. Iron chelators to starve the fungus of iron stores have been proposed using drugs such as deferiprone and deferasirox $[2,7]$.

\section{Conclusion}

A high index of suspicion for mucormycosis is necessary as less than 50 percent of cases are diagnosed antemortem [4]. This case is unique because mucormycosis was diagnosed antemortem and the bronchoscopic findings reveal a classic description of the damage Mucor species can inflict in susceptible patients. Although rare, mucormycosis can cause devastating effects in the immunocompromised host, as seen in this case. In this patient, surgical resection of the necrotic tissue would give this patient this best chance of survival. Resection was performed because it was felt she would have the best chance of survival at such a young age. Unfortunately, her disease was diffuse and she succumbed to her infection. Further research is needed to improve both diagnosis treatment of this troublesome disease. 
Citation: Heimes J, Zaheer S, Wallen J (2016) Bronchial Necrosis from Mucormycosis: A Case Report and Review. J Pulm Respir Med 6: 385. doi: 10.4172/2161-105X.1000385

Page 4 of 4

\section{References}

1. Merritt RE, Shrager JB (2012) Indications for surgery in patients with localized pulmonary infection. Thorac Surg Clin 22: 325-332.

2. Hamilos G, Samonis G, Kontoyiannis DP (2011) Pulmonary mucormycosis Semin Respir Crit Care Med 32: 693-702.

3. Garnacho-Montero J, Olaechea P, Alvarex-Lerma F, Alvarez-Rocha L, Blanquer J, et al. (2013) Epidemiology, diagnosis and treatment of fungal respiratory infections in the critically ill patient. Rev Esp Quimioter 26: 173-188.

4. Panigrahi MK, Manju R, Kumar SV, Toi PC (2014) Pulmonary Mucormycosis Presenting as Nonresolving Pneumonia in a Patient with Diabetes Mellitus. Respir Care 59: 1-5.

5. Patel A, Bishburg E, Nagarakanti S (2014) Mucormycosis in an HIV-infected renal transplant patient: A case report and review of the literature. Am J Case Rep 15: 74-78.

6. Ribes JA, Vanover-Sams CL, Baker DJ (2000) Zygomycetes in human disease Clin Microbiol Rev 13: 236-301.

7. Spellberg B, Edwards J Jr, Ibrahim A (2005) Novel perspectives on mucormycosis: pathophysiology, presentation, and management. Clin Microbiol Rev 18: 556-569.

8. Kontoyiannis DP, Lewis RE (2006) Invasive zygomycosis: update on pathogenesis, clinical manifestations, and management. Infect Dis Clin North Am 20: 581-607.

9. Ibrahim AS, Spellberg B, Avanessian V, Fu Y, Edwards JE Jr (2005) Rhizopus oryzae adheres to, is phagocytosed by, and damages endothelial cells in vitro. Infect Immun 73: 778-783.

10. Liu M, Spellberg B, Phan QT, Fu Y, Fu Y, et al. (2010) The endothelial cell receptor GRP78 is required for mucormycosis pathogenesis in diabetic mice. $J$ Clin Invest 120: 1914-1924.

11. Boelaert JR, de Locht M, Van Cutsem J, Kerrels V, Cantinieaux B, et al. (1993) Mucormycosis during deferoxamine therapy is a siderophore-mediated infection. In vitro and in vivo animal studies. J Clin Invest 91: 1979-1986.

12. Ibrahim AS, Gebermariam T, Fu Y, Lin L, Husseiny MI, et al. (2007) The iron chelator deferasirox protects mice from mucormycosis through iron starvation. J Clin Invest 117: 2649-2657.

13. Roden MM, Zaoutis TE, Buchanan WL, Knudsen TA, Sarkisova TA, et al (2005) Epidemiology and outcome of zygomycosis: a review of 929 reported cases. Clin Infect Dis 41: 634-653.

14. Reed C, Ibrahim A, Edwards JE Jr (2006) Deferasirox, an iron-chelating agent, as salvage therapy for rhinocerebral mucormycosis. Antimicrob Agents Chemother 50: 3968-3969.

15. Lee FY, Mossad SB, Adal KA (1999) Pulmonary mucormycosis: the last 30 years. Arch Intern Med 159: 1301-1309.

16. Chamilos G, Luna M, Lewis RE, Bodey GP, Chemaly R, et al. (2006) Invasive fungal infections in patients with hematologic malignancies in a tertiary care cancer center: an autopsy study over a 15-year period (1989-2003). Haematologica 91: 986-989.
17. Wahba $H$, Truong MT, Lei X, Kontoyiannis DP, Marom EM (2008) Reversed halo sign in invasive pulmonary fungal infections. Clin Infect Dis 46: 1733-1737.

18. Chamilos G, Marom EM, Lewis RE, Lionakis MS, Kontoyiannis DP (2005) Predictors of pulmonary zygomycosis versus invasive pulmonary aspergillosis in patients with cancer. Clin Infect Dis 41: 60-66.

19. Glazer M, Nusair S, Breuer R, Lafair J, Sherman Y, et al. (2000) The role of BAL in the diagnosis of pulmonary mucormycosis. Chest 117: 279-282.

20. Frater JL, Hall GS, Procop GW (2001) Histologic features of zygomycosis: emphasis on perineural invasion and fungal morphology. Arch Pathol Lab Med 125: 375-378.

21. Chamilos G, Lewis RE, Kontoyiannis DP (2008) Delaying amphotericin $\mathrm{B}$-based frontline therapy significantly increases mortality among patients with hematologic malignancy who have zygomycosis. Clin Infect Dis 47: 503-509.

22. Perfect JR (2005) Treatment of non-Aspergillus moulds in immunocompromised patients, with amphotericin B lipid complex. Clin Infect Dis 40: S401-S408.

23. Walsh TJ, Heimenz JW, Seibel NL, Perfect JR, Horwith G, et al. (1998) Amphotericin $\mathrm{B}$ lipid complex for invasive fungal infections: analysis of safety and efficacy in 556 cases. Clin infect Dis 26: 1383-1396.

24. Walsh TJ, Goodman JL, Pappas P, Bekersky I, Buell DN, et al. (2001) Safety, tolerance, and pharmacokinetics of high-dose liposomal amphotericin $B$ (AmBisome) in patients infected with Aspergillus species and other filamentous fungi: maximum tolerated dose study. Antimicrob Agents Chemother 45: 3487 3496.

25. Revankar SG, Hasan MS, Smith JW (2007) Cure of disseminated zygomycosis with cerebral involvement using high dose liposomal amphotericin $\mathrm{B}$ and surgery. Med Mycol 45: 183-185.

26. Dannaoui E, Meis JF, Loebenberg D, Verweij PE (2003) Activity of posaconazole in treatment of experimental disseminated zygomycosis. Antimicrob Agents Chemother 47: 3647-3650.

27. Dannaoui E, Meletiadis J, Mouton JW, Meis JF, Verweij PE, et al. (2003) In vitro susceptibilities of zygomycetes to conventional and new antifungals. J Antimicrob Chemother 51: 45-52.

28. Greenberg RN, Mullane K, van Burik JA, Raad I, Abzug MJ, et al. (2006) Posaconazole as salvage therapy for zygomycosis. Antimicrob Agents Chemother 50: 126-133.

29. van Burik JA, Hare RS, Solomon HF, Corrado ML, Kontoyiannis DP (2006) Posaconazole is effective as salvage therapy in zygomycosis: a retrospective summary of 91 cases. Clin Infect Dis 42: e61-e65.

30. Reed C, Bryant R, Ibrahim AS, Edwards J Jr, Filler SG, et al. (2008) Combination polyene-caspofungin treatment of rhino-orbital-cerebral mucormycosis. Clin Infect Dis 47: 364-371.

31. Tedder M, Spratt JA, Anstadt MP, Hegde SS, Tedder SD, et al. (1994 Pulmonary mucormycosis: results of medical and surgical therapy. Ann Thorac Surg 57: 1044-1050.

32. John BV, Chamilos G, Kontoyiannis DP (2005) Hyperbaric oxygen as an adjunctive treatment for zygomycosis. Clin Microbiol Infect 11: 515-517. 